\title{
First-Order Phase Transitions in Frustrated Spin Systems
}

\author{
Akihisa Koga, Akira Kawaguchi, Kouichi Okunishi* and Norio Kawakami \\ Department of Applied Physics, Osaka University, Osaka 565-0871 \\ * Department of Physics, Niigata University, Igarashi 2, Niigata 950-2181
}

(Received )

\begin{abstract}
We give a short review of our recent works on the first-order quantum phase transitions in frustrated spin chains with orthogonal-dimer structure. When the ratio of the competing antiferromagnetic exchange couplings is varied, a first-order transition occurs between the dimer phase and the plaquette phase, which is accompanied by the discontinuity in the spin excitation gap. We further show that strong frustration triggers the phase transitions in a magnetic field, which exhibit plateaus and jumps in the magnetization curve. The hole-doping effect is also addressed for the orthogonal-dimer chain with linked-tetrahedra structure. It is found that the competing antiferromagnetic interactions result in a firstorder metal-insulator transition upon hole doping.
\end{abstract}

\section{$\S 1 . \quad$ Introduction}

Recently antiferromagnetic quantum spin systems with strong frustration have been studied intensively. A typical example is $\mathrm{SrCu}_{2}\left(\mathrm{BO}_{3}\right)_{2}{ }^{1)}$ where the spin-1/2 magnetic $\mathrm{Cu}$ ions are located on the orthogonal-dimer lattice (the so-called ShastrySutherland model). ${ }^{2)}$ Various interesting phenomena were observed for this compound such as dispersionless triplet excitations, correlated hopping of two magnons, plateaus in the magnetization curve. It is suggested that novel properties in this compound are closely related to frustration due to the competing antiferromagnetic couplings. ${ }^{3), 4)}$ Besides such spin systems, metallic systems with frustration have also attracted much attention recently. For instance, it is suggested that heavy-fermion behavior observed in $\mathrm{LiV}_{2} \mathrm{O}_{4}{ }^{5)}$ may be caused by geometrical frustration inherent in the pyrochlore-lattice structure. ${ }^{6}$ )

One of the remarkable properties common in such frustrated systems is that strong frustration enhances the competition among several eigenstates with distinct character as a candidate for the ground state, giving rise to first-order quantum phase transitions when we vary the exchange couplings, the magnetic field, the chemical potential, etc. In order to address the role of geometrical frustration in quantum phase transitions, in this paper, we investigate a simple one-dimensional (1D) version of the frustrated spin model with orthogonal-dimer structure. By means of the series expansion technique, ${ }^{7)}$ the exact diagonalization (ED) and the density matrix renormalization group (DMRG), $\left.{ }^{8)}, 9\right)$ we demonstrate that strong frustration indeed causes a wide variety of first-order quantum phase transitions. 


\section{$\S 2$. Quantum spin system with orthogonal-dimer structure}

In this section, we study first-order transitions in the $1 \mathrm{D}$ orthogonal-dimer spin chain. ${ }^{10), 11)}$ The Hamiltonian we shall deal with is the standard $S=1 / 2$ antiferro-

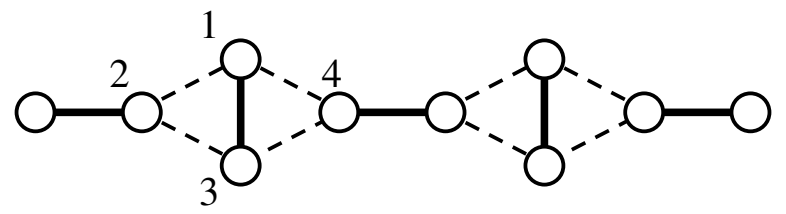

Fig. 1. Heisenberg spin chain with orthogonal-dimer structure. The solid (dashed) line represents the antiferromagnetic coupling $J\left(J^{\prime}\right)$.

magnetic Heisenberg model in a magnetic field $H, \mathcal{H}=\sum J_{i j} \mathbf{S}_{i} \cdot \mathbf{S}_{j}-H \sum S_{i}^{z}$, on the orthogonal-dimer chain shown in Fig. 1. We will use the normalized parameters $j=J^{\prime} / J$ and $h=H / J$ for convenience.

We first summarize the ground state properties of the orthogonal-dimer spin chain. ${ }^{10), 11)}$ The remarkable point characteristic of the orthogonal-dimer system is that a direct product of local dimer-singlet states indicated by the solid line in Fig. 1 is always an exact eigenstate of the Hamiltonian. Therefore, the dimer-singlet state should be the exact ground state up to a certain critical value of $j .{ }^{10}$ ) On the other

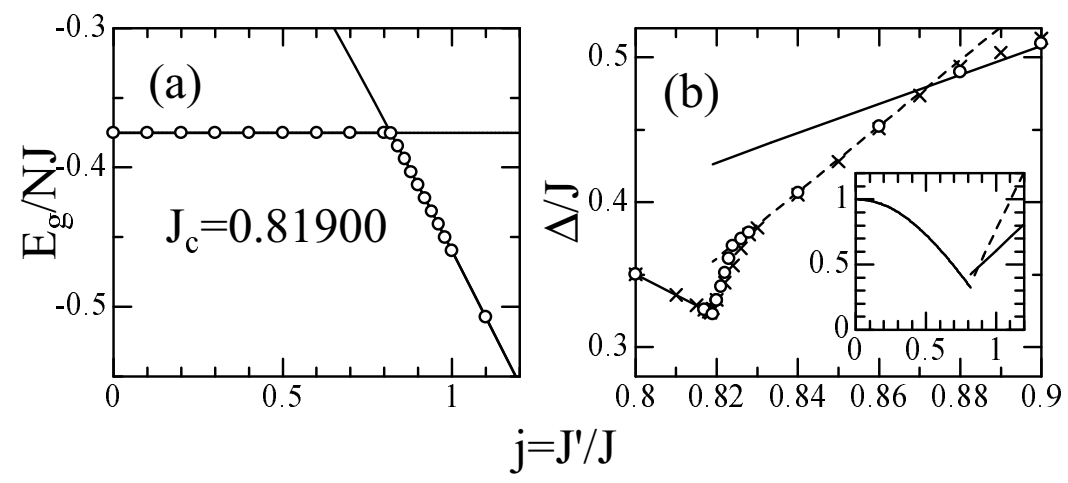

Fig. 2. (a) Ground state energy per site and (b) magnetic excitation energy $\Delta$ as a function of $j$ for the orthogonal-dimer chain. Crosses and open circles represent the results obtained for the finite chain of $N=16,24$ with periodic boundary conditions.

hand, in the large $j$ region, another singlet state is stabilized, which is adiabatically connected to the isolated plaquette-singlet denoted by the broken line in Fig. 1. ${ }^{12), 13)}$ As seen from Fig. 2 (a), a first-order quantum phase transition occurs between the dimer and the plaquette phases at the critical point, $j_{c}=0.81900$.

Let us now turn to the spin excitation spectrum. In the dimer phase $\left(j<j_{c}\right)$, the orthogonal-dimer structure forbids free hopping of a triplet excitation created over the local dimer state. ${ }^{2), 3)}$ On the other hand, in the plaquette phase $\left(j>j_{c}\right)$ there are two kinds of low-energy magnetic excitations. One is a dispersive triplet excitation, which is formed by simply breaking the plaquette singlet state. The 
corresponding excitation energy is shown as the solid line in Fig. 2 (b). The other is an unusual four-fold degenerate excitation shown as the dashed line, which is formed by breaking the plaquette singlet state first, and then making a local dimer state accompanied by two free spins. ${ }^{11)}$ Since the latter excitation becomes the lowestexcitation in the region $j_{c}<j<0.87$, as shown in Fig. 2, it plays an essential role for the phase transitions in the vicinity of the critical point, as will be shown below. In this way, the unusual four-fold degenerate excitation found here possesses intermediate properties between those typical for the dimer phase and the plaquette phase.

We have seen so far that several different kinds of low-energy excitations coexist in our frustrated model. It is thus interesting to see how these excitations affect the magnetization process. To this end, we focus here on the magnetization curve for the ratio of the exchange couplings $j=0.94$, where the system belongs to the plaquette phase. As shown in Fig. 3, the lowest excitation is a plaquette-triplet excitation (solid line), and another four-fold dispersionless excitation (broken line) lies slightly above it. The coexistence of two-kinds of distinct excitations influences
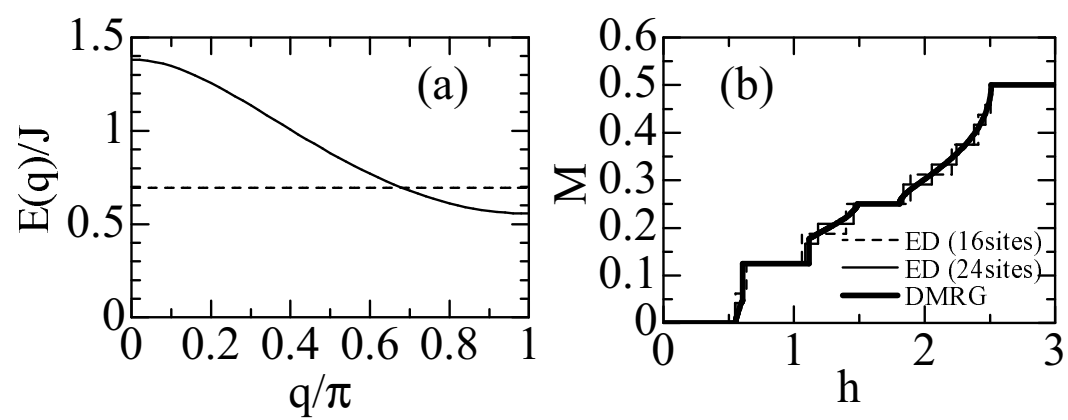

Fig. 3. (a) Dispersion relation calculate by the series expansion method ${ }^{11)}$ and (b) magnetization curve calculated by ED and DMRG for $j=0.94$.

the magnetization curve considerably. As $h$ increases beyond the critical field $\left(h_{c}=\right.$ $\Delta)$, the magnetization should develop with $M \sim\left(h-h_{c}\right)^{1 / 2}$, since it is dominated by the triplet excitation whose dispersion relation is quadratic near the bottom $(q=\pi)$. With slight increase in $h$, however, the magnetization suddenly jumps to the $1 / 4$ plateau. This first-order transition results from the coexistence of two distinct levels in low-energy excitations. Therefore, it is instructive to remark that the $1 / 4$-plateau is not generated by the crystallization of the lowest triplet excitation, but of the four-fold degenerate excitation. Beyond the 1/4-plateau, we again encounter another first-order phase transition accompanied by a jump in $M$.

As seen in this section, nontrivial behavior shows up reflecting the competing interactions in the present model. We wish to observe below what happens for such 1D spin systems, when we introduce the charge degree of freedom, which is important to discuss a metallic system with strong frustration. 


\section{$\S 3 . \quad$ Correlated electron system with strong frustration}

In this section, we extend our discussions to a hole-doped system. ${ }^{14)}$ We here employ another orthogonal-dimer model ${ }^{15}$ ) shown in Fig. 4. A nice feature in this model is that it can describe not only the orthogonal-dimer spin system but also the linked-tetrahedra system, which plays an important role for understanding low-energy properties of the pyrochlore system. In the undoped case, a first-order quantum phase transition between the dimer phase and the plaquette phase occurs at the critical point $\left.j_{c}=0.71 .{ }^{15)}, 16\right)$

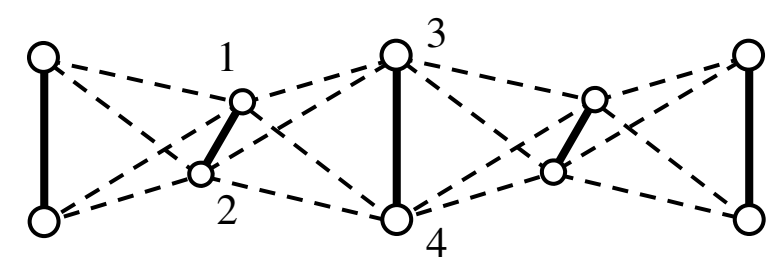

Fig. 4. Orthogonal-dimer spin chain with liked-tetrahedra structure. The solid and the dashed lines correspond to the parameters $J(t)$ and $J^{\prime}\left(t^{\prime}\right)$, respectively.

We shall deal with a hole-doped system by introducing the $t$ - $J$ model with electron hopping, $t$ and $t^{\prime}$, and clarify how frustration affects the nature of the metalinsulator transition. Let us first consider the hole-doping effects on the dimer phase. For simplicity, we set $t / J=t^{\prime} / J^{\prime}=4.0$. In Fig. 5 (a), the electron density $n$ calcu-
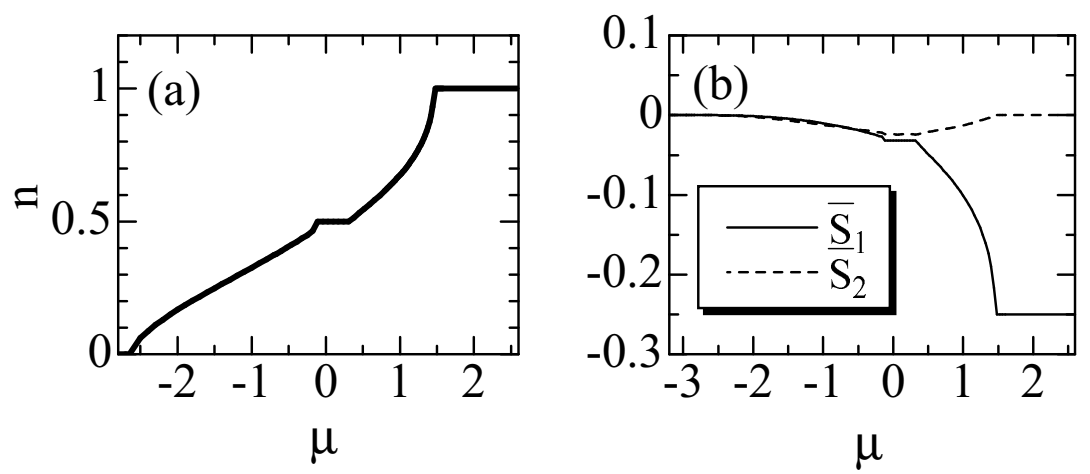

Fig. 5. (a) Electron density $n$ and (b) short-range correlation $\bar{S}_{\alpha}$ as a function of the chemical potential $\mu$ for $j=0.4$ (dimer phase).

lated by DMRG is shown as a function of the chemical potential $\mu$ for $j=0.4$. In this figure, $n=1$ corresponds to half-filling at which the system is in the insulating phase with singlet-dimer state. It is seen that hole-doping smoothly drives the system to a metallic phase. At quarter filling $(n=1 / 2)$, we encounter another insulating phase, which is stabilized by the formation of a CDW state.

In order to see the nature of spin correlations in the metallic phase, we compute the short-range spin correlation functions in the rung and chain directions, which 
are defined as (see Fig. 4),

$$
\begin{aligned}
& \bar{S}_{1}=\frac{1}{2}\left(<S_{1}^{z} S_{2}^{z}>+<S_{3}^{z} S_{4}^{z}>\right), \\
& \bar{S}_{2}=\frac{1}{4}\left(<S_{1}^{z} S_{3}^{z}>+<S_{1}^{z} S_{4}^{z}>+<S_{2}^{z} S_{3}^{z}>+<S_{2}^{z} S_{4}^{z}>\right) .
\end{aligned}
$$

In Fig. 5 (b), the spin correlation functions calculated by DMRG are shown as a function of the chemical potential $\mu$. Note that we have $\bar{S}_{1}=-1 / 4$ and $\bar{S}_{2}=0$ at half filling, which accord with those expected for the isolated dimers. It is seen that the spin correlation along the rung, $\bar{S}_{1}$, rapidly decreases down to $-1 / 4$ as the system approaches half filling from the metallic side. On the other hand, $\bar{S}_{2}$ becomes almost zero along the chain direction, reflecting the fact that the system is an assembly of decoupled dimers at half filling. Therefore, near half filling, the metallic state is considered as a resonating state composed of such dimer pairs.

In contrast to the dimer phase, strong frustration dramatically changes the nature of the metal-insulator transition in the plaquette phase. In Fig. 6 (a), we show
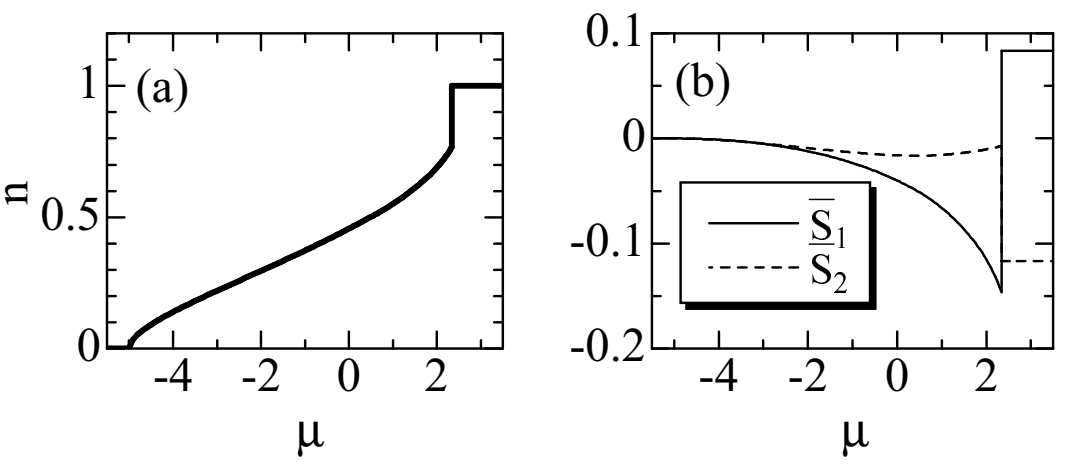

Fig. 6. (a) Electron density $n$ and (b) short-range correlation $\bar{S}_{\alpha}$ as a function of $\mu$ for $j=1.0$ (plaquette phase).

the electron density $n$ as a function of $\mu$ for $j=1.0$, where the system is in the plaquette-singlet phase at half filling. It should be noticed that as $\mu$ decreases from half filling $(n=1)$, a first-order phase transition suddenly occurs to the metallic phase at $\mu \simeq 2.2$, which is accompanied by the discontinuity in the electron density. To clarify the nature of the metal-insulator transition in more detail, we again discuss the short-range spin correlation function [see Fig. 6 (b)]. In the insulating phase $(n=1)$ for $\mu>2.2$, the ground state is in the plaquette-singlet phase, which is characterized by $\bar{S}_{1}>0$ and $\bar{S}_{2}<0$ in accordance with the numerical results. On the other hand, once the first-order transition occurs, both of $\bar{S}_{1}$ and $\bar{S}_{2}$ have negative values in the metallic phase. Note that $\bar{S}_{1}$ decreases with negative values while $\bar{S}_{2}$ gets very small as the system approaches half-filling from the metallic side. This implies that the dimer correlation along the rung is largely enhanced in the metallic phase close to half filling. This dimer-dominant state competes with the plaquette-singlet state realized at half filling, triggering the first-order phase transition. 


\section{$\S 4$. Summary}

We have investigated the first-order quantum phase transitions in antiferromagnetic spin chains with orthogonal-dimer structure. It has been clarified that there appears rich structure in the excitation spectrum, which is caused by strong frustration. Such nontrivial excitations determine the nature of the quantum phase transitions, when we change the interactions, the magnetic field, etc. We have also studied the hole-doping effect on another orthogonal-dimer chain with linked-tetrahedra structure. It has been shown that the first-order metal-insulator transition is triggered by strong geometrical frustration. We have checked in a preliminary calculation that such a first-order metal-insulator transition also appears in the model of $\S 2$, implying that it may be common in this class of the orthogonal-dimer models.

\section{Acknowledgements}

This work was partly supported by a Grant-in-Aid from the Ministry of Education, Science, Sports and Culture of Japan. A part of computations was done at the Supercomputer Center at the Institute for Solid State Physics, University of Tokyo and Yukawa Institute Computer Facility. A. Kawaguchi is supported by the Japan Society for the Promotion of Science.

\section{References}

1) H. Kageyama, K. Yoshimura, R. Stern, N. V. Mushnikov, K. Onizuka, M. Kato, K. Kosuge, C. P. Slichter, T. Goto and Y. Ueda: Phys. Rev. Lett. 823168 (1999).

2) B. S. Shastry and B. Sutherland: Physica 108B 1069 (1981).

3) S. Miyahara and K. Ueda: Phys. Rev. Lett. 82, 3701 (1999).

4) A. Koga and N. Kawakami: Phys. Rev. Lett. 84, 4461 (2000); C. Knetter, A. Bühlet, E. Müller-Hartmann and G. S. Uhrig: Phys. Rev. Lett. 85, 3958 (2000); T. Momoi and K. Totsuka, Phys. Rev. B 613231 (2000); Y. Fukumoto: J. Phys. Soc. Jpn. 69, 2755 (2000); G. Misguish, Th. Jolicoeur and S. M. Girvin, Phys. Rev. Lett. 87097203 (2001); C. H. Chung, J. B. Marston and S. Sachdev, Phys. Rev. B 64134407 (2001).

5) S. Kondo, D. C. Johnston, C. A. Swenson, F. Borsa, A. V. Mahajan, L. L. Miller, T. Gu, A. I. Goldman, M. B. Maple, D. A. Gajewski, E. J. Freeman, N. R. Dilley, R. P. Dickey, J. Merrin, K. Kojima, G. M. Luke, Y. J. Uemura, O. Chmaissem and J. D. Jorgensen, Phys. Rev. Lett. 78, 3729 (1997).

6) H. Kaps, N. Büttgen, W. Trinkl, A. Loidl, M. Klemm and S. Horn, J. Phys. Condens. Matter 13, 8497 (2001).

7) M. P. Gelfand and R. R. P. Singh, Adv. Phys. 49, 93 (2000).

8) S. R. White, Phys. Rev. Lett. 69, 2863 (1992); Phys. Rev. B 48, 10345 (1993).

9) T. Nishino and K. Okunishi, J. Phys. Soc. Jpn. 63, 4084 (1995); Y. Hieida, K. Okunishi and Y. Akutsu, Phys. Lett. A 233, 464 (1997).

10) N. B. Ivanov and J. Richter, Phys. Lett. 232A, 308 (1997); J. Richter, N. B. Ivanov and J. Schulenburg, J. Phys. Condence Matt. 10, 3635 (1998); J. Schulenburg and J. Richter, Phys. Rev. B 65, 054420 (2002).

11) A. Koga, K. Okunishi and N. Kawakami, Phys. Rev. B 62, 5558 (2000).

12) N. Kato and M. Imada, J. Phys. Soc. Jpn. 64, (1995) 4105.

13) A. Koga, S. Kumada, N. Kawakami and T. Fukui, J. Phys. Soc. Jpn. 67, 622 (1998).

14) M. Ogata, M. U. Luchini, T. M. Rice, Phys. Rev. B 44 (1991) 12083.

15) M. P. Gelfand, Phys. Rev. B 43, 8644 (1991).

16) A. Honecker, F. Mila and M. Troyer, Eur. Phys. J. B, 15 227,(2000). 